\title{
Meta-View of Blockchain Technology Tensions in Organizational Implementation and Use
}

\author{
Nadine Kathrin Ostern \\ Marburg University, Germany \\ nadine.ostern@wiwi.uni-marburg.de
}

\author{
Guido Perscheid \\ Frankfurt School of Finance and Management \\ Germany,g.perscheid@fs.de
}

\begin{abstract}
Blockchain inherits tensions that depend on its infrastructure design as well as distinctive features of, among others, smart contracts and storage mechanisms. Studies propose coping strategies for companies that deal with blockchain-related tensions, but so far suggestions are characterized by context- and casespecificity. This paper proposes a meta-view on tensions arising from the implementation and usage of blockchain in organizational contexts. A structured literature review is conducted to condense existing insights from the literature to a meta-view on blockchain-related tension. A framework provides insights into different types of tensions. The framework has two aims: (1) providing a foundation to jointly build insights and IS theorization on blockchain tensions without being case- and context-sensitive. (2), it provides food for thought for companies that want to implement or use blockchain, which serves as first step toward developing recommendations that guide companies through identifying and managing tensions arising from blockchain implementation and usage.
\end{abstract}

\section{Introduction}

Every company faces the problem of how to make progress on seemingly conflicting objectives at the same time. When it comes to emerging technology usage, efforts to build for a tomorrow sometimes distract from producing results today [1]. This especially holds for mature firms that need to balance tensions resulting from exploring new developments for future performance, while exploiting existing information systems capabilities to generate sufficient value in the short term [2]. Blockchain technology confronts companies with precisely such a challenge. Allowing for disintermediation and the reduction of transaction costs (through the realization of contracts via smart contracts), blockchain enables significant efficiency gains and cost-saving potentials in the future [3,4]. Even beyond, the capabilities of smart contracts might enable far-reaching process automation and restructuring of sharing business models through new forms of collaborative information systems [5,6]. At the same time, the openness of the underlying peer-to-peer network poses some significant challenges in the now. Designed as an open blockchain, the question arises as to how the shared innovation can guarantee that all participants capture value [7]. If a blockchain is (partially) privatized, dependency relationships of certain participants and the question of data sovereignty arise [8]. Companies that want to develop or use blockchain must therefore be very clear about the potential tensions that can emerge when utilizing blockchain technology.

This paper asks, what are the tensions that arise in the implementation and use of blockchain technology for businesses and how can we conceptualize them? A structured literature review is used for providing a metaview that transfers existing tensions into a more abstract framework, helping to abstract from case-specific tensions. This is an important step towards visualizing and discussing common blockchain tensions that companies need to consider when planning the implementation or using blockchain technology.

By answering the research question, we provide a meta-view of previously identified and discussed tensions. This serves to synthesize existing findings and provides a basis for further discussion and theory development on blockchain's implementation. At the same time, the results have practical relevance as these provide initial food for thought on the application of the technology and the potential tensions that may arise from it. We provoke thought by comparing and highlighting the relevance of blockchain-specific tensions compared to general tensions all companies face identified by Dodd and Favaro [1].

The remainder of this paper is structured as follows: Section 2 presents related work on blockchain technology and interpreting tensions using a paradox lens. Case- and context specificity is pointed out as one characteristic of many tension studies that hinder discussing them on a more abstract level, fostering the development of specific theory and strategies for companies in implementing and using blockchain 
technology. Next, the literature review and data analysis are described in the third section. Section 4 is concerned with presenting and exemplifying the results of the structure literature review, depicting the tensions framework for blockchain technology. The results are discussed in section 5. Eventually, section 6 provides a conclusion and outlook on future work.

\section{Related Work}

This section introduces blockchain technology and its distinguishing features. Then, related work on paradox theory, which is often used to interpret and understand tensions in different IS contexts, is presented.

\subsection{Blockchain Technology}

Blockchain technology is a distributed ledger technology in the form of a distributed transactional database, secured by cryptography, and governed by consensus mechanisms [9]. Thereby, blockchain enables the secure and immutable storage of transactional information. Besides, the most essential feature of blockchain might be so-called smart contracts, which allow automating agreements written as programs on the blockchain. In general, this unlashes the possibility to create reliable, trust-free distributed record systems, that may fundamentally change the way how we organize processes $[9,10]$.

What is important, however, is that blockchain is characterized by a high degree of flexibility when it comes to the actual components of the technologies and thus to the properties of openness, access authorization, and revisability - to name just a few. Thus, scholars typically differentiate between different types of blockchain [11], i.e.:

- Public blockchain: all nodes are allowed to read and propose new blockchain entries,

- Private blockchain: only preregistered nodes, granted by a central authority, are allowed to read and propose new blockchain entries,

- Hybrid blockchain or consortium: only nodes in the consortium are allowed to read blockchain and propose blockchain entries.

Based on what kind of blockchain type, various design issues emerge that are at the core of blockchain tensions. For instance, public blockchains offer various benefits in terms of data interchange, transparency, and accountability, while data security and confidentiality might be at risk [12]. In contrast, employing a private blockchain may resolve data security and confidentiality risks, but functionalities of the original open blockchain (as in the first use case of bitcoin) may be lost, and thus efficiency gains as well as the incentive for companies to use this technology $[13,14]$.

While companies may have different motivations for using blockchain technology (e.g., marketing purposes, experience, standardization of interfaces [8]), they should be aware of the underlying tensions - not only those arising from different types of blockchain, but also other design decisions. To do this, we will first look at the broader literature on tensions and then move on to examine blockchain's tensions.

\subsection{Paradox Theory and Tensions}

Information system scholars increasingly apply a paradox lens to understand tensions arising from information technology implementation and use. The paradox perspective, thereby, has two components: a contradiction between (at least) two propositions (the tension), and the resolution of the tension [15].

The paradox lens has also been applied when it comes to blockchain implementation, e.g., in the context of car-related ecosystems [15] or social networks [16]. What these studies all have in common, however, is that they identify tensions and coping mechanisms for very specific applications of blockchain. This approach can also be observed in the broader management literature, in which studies have been pointed out to be largely anecdotal or single case studies that offer primarily contextual approaches [17].

While these are undoubtedly important contributions, IS research is also in need for studies providing a more abstract view on tensions - detached from the case-specific challenges of blockchain implementations - which can and will help to effectively manage tensions arising from enable incremental innovation and exploring opportunities through radical innovation at the same time [17]. Notably, the metaview is expected to build common grounds to build a common theory on tensions in blockchain technology, giving scholars and organizations guidance in how to better identify, understand and manage potentially emerging tensions from specific design decisions in the course of the technology's implementation and use.

\section{Literature Review and Tensions}

To obtain a meta-view of the tensions associated with blockchain, we conducted a structured literature review [20]. Using the search term: (blockchain* OR "distributed ledger") AND (tension* OR problem* OR traction* OR pressure* OR challenge*) we searched for peer-reviewed papers that intentionally address tensions and related concepts pertaining to blockchain implementation or use in an organizational context. The search was conducted in the databases of the leading IS 
journals, i.e., Basket of Eight IS Journals as well as the AIS eLibrary. The search resulted in a total number of 156 articles. Aiming to also include relevant articles from other databases, such as the Harvard Business Review, we conducted a second search using the same search term on Google Scholar, allowing us to add 7 further papers to our sample.

The resulting sample of 163 papers was then screened for exclusion and inclusion criteria. We excluded abstracts only papers, research in progress papers, panel and workshop formats, and editorials, leaving us with a total of 84 papers. The remaining papers were read and excluded when they only referred to tensions as a further explanatory factor or briefly mentioned them without further elaboration, reducing the sample to 51 papers. Based on the remaining papers, we performed a forward and backward search, allowing us to identify and add 5 more papers to our final sample of 56 papers. The 56 papers were then analyzed using open, axial, and selective coding. The coding was performed by both authors individually. After each step of coding (open, axial, and selective coding) the results were discussed until consensus was found. For open coding, articles were read and any insight in the text that seemed relevant was highlighted. Open coding led to a first set of identified and labeled sets of concepts and insights supported by the papers [18]. Axial coding then engages in building categories, thereby grouping concepts into main categories and, potential, subcategories. Axial coding led to the main themes of the analyzed studies' [18]. Eventually, selective coding was used to integrate and refine the categories that were identified $[18,19]$.

All three coding stages were performed at least once for each paper, until theoretical saturation was reached, i.e., no new categories and themes were to be identified from the articles [20]. A logbook was used to document relevant decisions and information that influenced the coding procedure to ensure transparency and comprehensibility of the coding process [18].

\section{Findings}

In the course of the literature analysis, 6 selective codes and 12 axial codes were identified representing the main tensions and categorization of these as summarized in Figure 1. In particular, throughout the literature review, two meta-characteristics of blockchain-related tensions were obvious, i.e., whether the tensions are controllable or manageable. Controllable tensions emerge from specific design decisions on the blockchain infrastructure that is in line with a companies' overall strategic goals. In contrast, manageable tensions, are tensions that emerge independent of design choices on the infrastructure level that need to be considered mostly in every case of blockchain design. Thus, companies need to manage them through implementation and usage, eventually applying particular coping strategies. To better comprehend this distinction, related categories of controllable and manageable tensions are explained and exemplified in the following. Due to the meta-view, the descriptions abstract from concrete cases and discuss and interpret controllable tensions along with the two opposing design decisions, i.e., public vs. private blockchain. Controllable and manageable tensions are both discussed in light of the vulnerabilities they imply, which must ultimately be resolved through coping strategies. With the objective of improved clarity and transparency, we listed the sources representing the result of our literature study consequently providing the foundation for the development of our framework and discussion section in Table 1.

\begin{tabular}{|c|c|}
\hline Tension areas & Identified literature \\
\hline Environmental Positioning & $\begin{array}{l}\text { Bazarhanova et al. 2019; Beck et al. 2018; Berente et al. 2016; Ciborra et al. 2020; Hoss et al. 2021; } \\
\text { Furlonger/Uzureau2019; Norstrom/Lindman 2020; Ostern et al. 2020; Viriyasitavat/Hoonsopon 2019 }\end{array}$ \\
\hline Organizational Alignment & $\begin{array}{l}\text { Bauer et al. 2019; Beck et al. 2018; Bazarhanova et al. 2019; Bogusz/Morisse 2018; Chen et al. 2021; Gordon/Catalini 2018; } \\
\text { Iansiti/Lakhani 2017; Norstrom/Lindman 2020; Renwick/Gleasure 2021; Schweizer et al 2017; Villareal } 2012\end{array}$ \\
\hline Value Discipline & Holotiuk/Moormann 2018; Montealegre et al. 2019; Morkunas et al. 2019; Ostern et al. 2020 \\
\hline Integration Model & Bazarhanova et al. 2019; Böhmeet al. 2015; Chen et al. 2021; Morkunas et al. 2019; Naerland et al. 2017; Perscheid et al. 2020 \\
\hline Trust Model & $\begin{array}{l}\text { Andersen/Bogusz 2017; Auinger/Riedl 2018; Beck et al. 2016; Betzwieser et al. 2019; Dodd/Favaro 2006; Glaser et al. 2019; Ostern } \\
\text { 2018; Renwick/Gleasure 2021; Zhao et al. 2018; Zavolokina et al. } 2019\end{array}$ \\
\hline Accountability Model & $\begin{array}{l}\text { Beck et al. 2018; Currie et al. 2019; Fabian et al. 2016; Ostern/Riedl 2020; Reid/Harrigan 2019; Rieger et al 2019; Scholl et al. 2020; } \\
\text { Yin et al. } 2019\end{array}$ \\
\hline $\begin{array}{l}\text { Tension area 1: } \\
\text { Growth vs. Profitability }\end{array}$ & $\begin{array}{l}\text { Anriopoulos/Lewis 2008; Coblenz et al. 2019; Dodd/Favaro 2006; Furlonger/Uzureau2019; Montealegre et al. 2019; Müller et al. } \\
\text { 2020; Ølnes et al. 2017; Riedmann-Streitz 2018; Wan et al. } 2018\end{array}$ \\
\hline $\begin{array}{l}\text { Tension area 2: } \\
\text { Long term vs. Short term }\end{array}$ & $\begin{array}{l}\text { Bauer et al. 2019; Cumming et al. 2019; Dodd/Favaro 2006; Montealegre et al. 2019; Ostern et al. 2020; Viriyasitavat/Hoonsopon } \\
2019\end{array}$ \\
\hline $\begin{array}{l}\text { Tension are 3: } \\
\text { Whole vs. Parts }\end{array}$ & Al-Breiki et al. 2020; De Filippi et al. 2020; Dodd/Favaro 2006 \\
\hline
\end{tabular}

Table 1. Identified literature referring to respective tension areas 


\subsection{Controllable Tensions}

Controllable tensions were identified as tensions concerned with corporate decisions regarding a companies' environmental positioning and organizational alignment [11,21-23]. Environmental positioning refers to companies' decision to maintain and participate in a public blockchain associated with the goal of participatory innovation and business value capture versus a private blockchain that implies focusing on competitive advantages through technology leadership. This tension (i.e., cooperation vs. competition) arises from the initial openness of blockchain's infrastructure (among others, apparent through consensus protocols for joint verification [24]) that needs to be balanced and aligned with the logic of the organization and its demands [22,23,25]. Thus, implementing a private blockchain may "drifts" away from the original idea of its use and the organization may lose control of it $[22,26]$. Notably, private blockchains may lead to a loss of value that might be cocreated in consortia or a fully open blockchain ecosystem [3], while at the same time it allows companies to control who captures value (e.g., through the use of data generated and stored on a blockchain) in a distributed infrastructure [8]. While the reversed logic applies to original applications of blockchain, i.e., public blockchains, companies must weigh these vulnerabilities in the light of their overall organizational strategy and positioning.

It is necessary to make an informed choice between these two propositions (or to find a compromise to cope with these propositions) as they have a significant impact on organizational alignment [27]. Organizational alignment is a tension that arises from the divide between aiming at primarily external value networks or internal value creation and capture (while these are to be understood as two not mutually exclusive extreme positions) [28-30]. Organizational alignment refers to the alignment of blockchain's infrastructure with internal and/or inter-corporate business processes that may lead to the creation of external value networks [27]. While reliance on external value networks implies possible effects on internal operational efficiency (e.g., through speed loss and dependency relationships) $[31,32]$, the internal value might limit the democratization of sensemaking on publicly available data that can foster distributed innovation and technological mashups [33]. Companies need to

consider organizational alignment as they therefore either tend to focus on achieving project goals and satisfying customer needs through internal value versus the initiative to probing new ideas through value networks to surface novel opportunities [34].

Since these tensions are strongly influenced by the initial infrastructure design or arise during a blockchain application (typically, each blockchain is highly centralized at the beginning of its use until a critical mass is established), we call them controllable. We will now move on to tensions that are to some extent independent of the initial design decisions, i.e., tensions that companies need to manage throughout blockchain implementation, adaptation, and its usage.

\subsection{Manageable Tensions}

Manageable tensions are challenges that are largely independent of the design decisions (public, private consortium blockchain) of companies about the setup of the blockchain infrastructure. They arise from the (partially) distributed nature of the blockchain. Therefore, these tensions cannot be controlled, but they

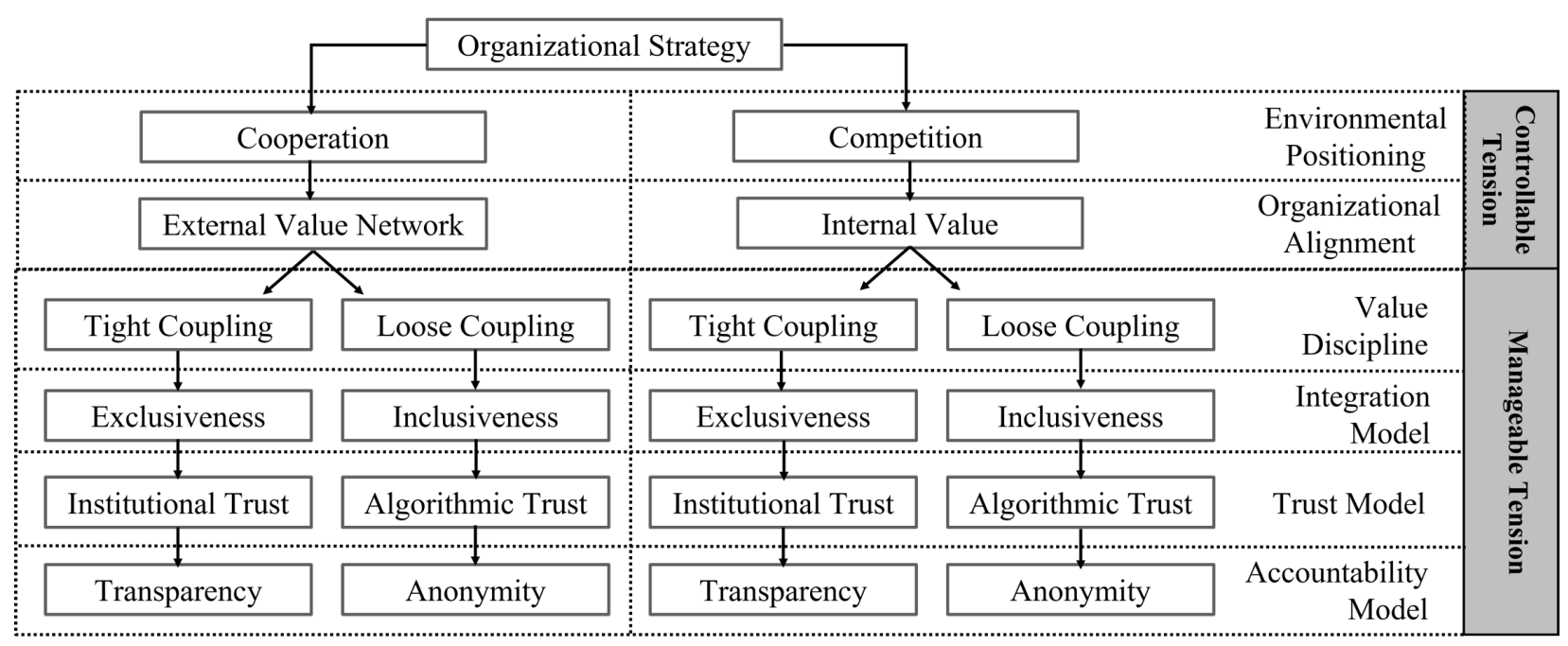

Figure 1. Framework of Blockchain Tensions 
can be actively managed. In the run-up to the implementation and use of blockchain, companies must therefore not only consider the strategic orientation and alignment with intra- and inter-organizational processes, but also, among other things, weigh up the traceability and anonymity, the exclusivity or inclusivity, and the form of trust. These manageable tensions are explained in the following.

The value discipline refers to tensions that relate to what companies want to achieve with a blockchain solution [35]. While this discipline seems similar to organizational alignment, the focus of the value discipline is not on alignment to intra- or interorganizational processes (and thus primarily on value capture), but on aligning value creation with the desires of a company's internal and external customers [36]. Tight coupling refers to the alignment of value creation with customer needs and demand fulfillment (e.g., greater traceability and transparency or higher returns by reducing unnecessary friction in customer processes). Loose coupling means that a company partially detaches itself from the customer's wishes and proposes something that goes beyond customercentricity. An example of the latter is financial service providers releasing free blockchain networks for peerto-peer lending to the unbanked to turn them into potential future customers [3]. While the former allows maximizing the existing potential of value creation, in loose coupling the company goes beyond risking failure and offering an infrastructure or service for which there is - at the moment - little demand. Independent of the infrastructure design, companies thus need to determine the degree of innovation and novelty in the application of blockchain, which is a tension with advantages and disadvantages that are often observed in the application of new technologies [37].

The integration model refers to tensions related to the openness of the blockchain application to other participants, i.e., whether an inclusive or exclusive approach is pursued. While both characteristics are possible in different configurations (competitive or cooperative), competitive models tend towards exclusivity, while cooperative approaches mainly promote inclusivity [21,38]. Exclusivity implies that participation and involvement in a blockchain are regulated. This might happen, among others, with access or validation rights that are granted through the consensus protocol, smart contracts as well as general entry barriers for individuals or companies that want to participate in the peer-to-peer network) [39,40]. Exclusivity thereby might range from strong exclusivity (e.g., intra-company blockchain applications) to mixed forms, such as company networks (i.e., consortia). The integration model yields the tension whether a company has a profit emphasis (i.e., focus on exclusivity) or a breakthrough emphasis (i.e., focus on inclusivity). In the latter case, the likelihood of innovative process and business model changes is more likely [32]. Hence, while the question of access and readability of blockchain entries is often discussed in the literature as a purely governance-related or security issue [40], it may also have implications for management trade-offs between growth and profitability $[1,32,36]$.

Trust in blockchain is a frequently addressed topic that extends along with the tensions of trust in institutions or the participants of a blockchain solution and trust in the blockchain code $[41,42]$. As in the other cases, compromises and mixed forms are possible; the propositions mentioned reflect the extreme forms $[15,43,44]$. Companies need to be aware of the tensions between these forms of trust and understand the consequences of paths between the different trust models [45]. For example, trust within an organization can significantly change the corporate culture. While trust in participants can, in the worst case, lead to a divergent cultural identity (e.g., divisions that use a blockchain solution and those that do not) and a culture of blaming or finger-pointing. In contrast, trust in code creates a sort of neutrality [1]. Trust in codes requires that companies agree on universally applicable rules and metrics that are tied to smart contracts [46]. This can lead to tensions in organizations a priori to the implementation and use of blockchain. Therefore, companies need to consider this trade-off very carefully and define the trust model prudently to anticipate downstream or upstream effects $[29,45,47]$.

Eventually, the accountability model was identified as a further area of tension in the IS literature [48,49]. The accountability model is strongly linked to compliance aspects in the organization, as the accountability model is responsible for whether a company allows strong transparency of logged records and responsibilities on the blockchain or anonymity resp. pseudo-anonymity [50,51]. From a management perspective, transparency allows for a democratization of validation and participation, which allows for the involvement of the entire organization and thus for complete decentralization. Anonymity may come at the cost of participation, as employees or other companies may not want to or be able to participate in a blockchain solution with an anonymous model. In particular, anonymous solutions make companies or company networks potentially more vulnerable to fraud or misuse of data and manipulation [11,51]. Additionally, companies might face several regulatory issues depending on their accountability model, since blockchain's properties (e.g., the irreversibility of data on the blockchain) are conflicting with laws, such as the EU General Data Protection Regulation (GDPR) [52]. However, as scholars have identified ways to comply 
with regulations such as GDPR when offering blockchain-based applications [53,54], we see the major issue lying in the agreement of all parties participating in the blockchain to the accountability model and associated business risks.

\section{Discussion}

The presented framework identifies the different core areas where tensions can arise during blockchain implementation and use and distinguishes between controllable and manageable tensions. The distinction and the attention to these differences and core areas should assist companies in the future to anticipate and better understand tensions arising from blockchains usage in corporate contexts. In particular, however, this section of the paper aims to relate these partly technical issues (i.e., the design and configuration of blockchain features) to general tensions of a company as discussed by [1]. This allows us to frame blockchain-related tensions in the context of recurring business considerations and to identify their relevance and contribution to each of the general tensions.

Scholars have identified three overarching tensions areas every company faces, i.e., (1.) profitability vs. growth, (2.) short term vs. long term, (3.) whole vs. parts [1]. These tensions must not require a unique strategy but represent a constant oscillation between two extreme propositions. In other words, tensions represent a pendulum to which companies are constantly exposed [1]. Blockchain tensions are discussed and interpreted along with these major tension areas in the following, acknowledging that the salience of tensions may change during the development and implementation of blockchain. Figure 2 summarizes the interpretation of

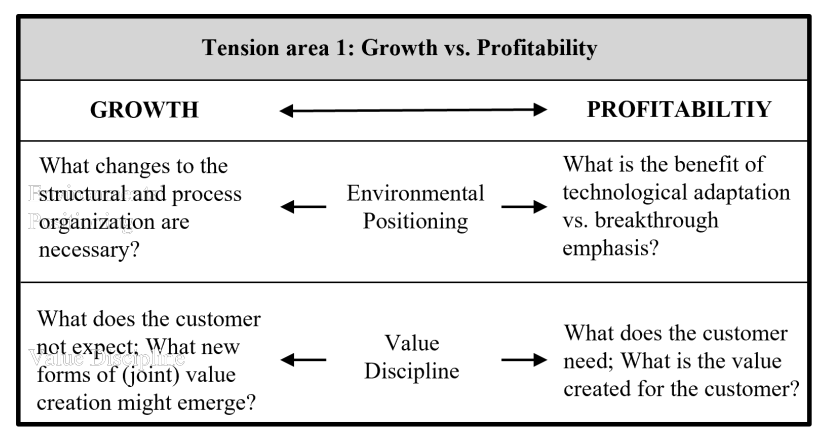

\begin{tabular}{|l|l|}
\hline \multicolumn{2}{|c|}{ Tension area 3: Whole vs. Parts } \\
\hline WHOLE & \multicolumn{1}{|c|}{ PARTS } \\
\hline $\begin{array}{l}\text { What standards and } \\
\text { recognized key indicators } \\
\text { must be agreed upon to } \\
\text { enable an algorithmic trust? }\end{array} \quad$ Trust Model $\longrightarrow \begin{array}{l}\text { How can trust be } \\
\text { established btw. selected } \\
\text { users; what does this } \\
\text { require from participants? }\end{array}$ \\
\hline
\end{tabular}

blockchain tensions along with these overall corporate tensions and proposes guiding questions for companies in identifying suitable coping strategies.

Tension area 1 - Profitability vs. growth: Companies have been observed to swing between growth push and productivity push, whereas in difficult times companies tend to give priority to reducing costs. In contrast, companies focus on growth in boom times [1], which might lead to a loss of control over costs as well as eroding margins. Once the focus shifts back to profitability, companies fall short on top-line growth, leading to a growth orientation again. At best, companies manage to balance these tensions, i.e. what we call organizational ambidexterity $[17,37]$.

If companies want to balance aspects of profitability and growth, they must consider the tension of environmental positioning and value discipline. In the context of environmental positioning, companies should particularly consider the benefits of a completely open blockchain and thus the possibility of participatory and distributed innovation vs. control over value capture, when deploying a private blockchain. The use of a (partially) private blockchain can promote the profitability of a company. Operators of private and semi-private blockchains have control over data and can lock their members into the solution for the long term through service contracts, creating not only a platform dependency but also a technology dependency [8]. This promotes the long-term profitability of the company.

On the other hand, the use of an open or semi-open blockchain and, thus focusing on cooperation, potentially leads to distributed innovation and growth through the emergence of new business models and collaborative processes $[14,55]$. It is important to

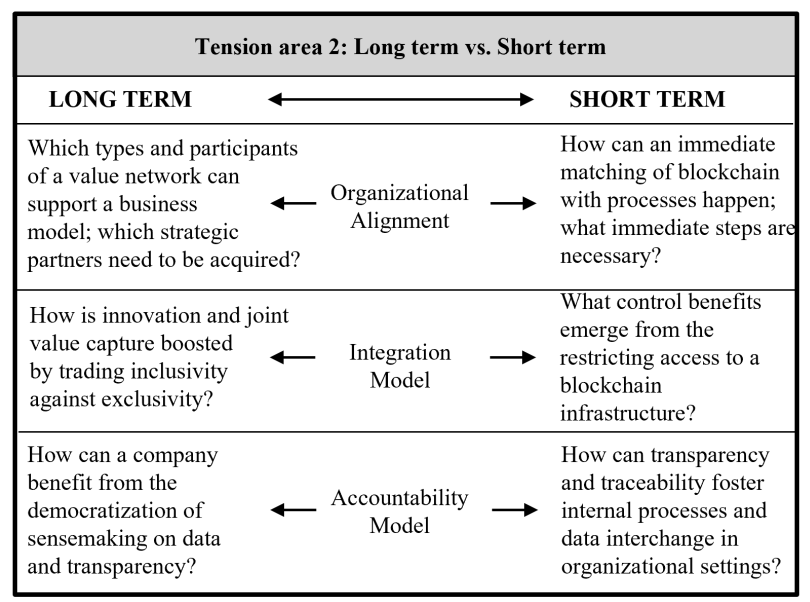

Table 2. Guiding questions to address blockchain tensions in organizations 
understand, that most companies never resolve the profitability vs. growth tension - it is like a balloon being squeezed at one end and expanding at another. Yet, a successful strategy to reconcile is to focus on the customer benefit of a blockchain application [1], leading us to the discussion of the role of the value discipline to manage tension area 1 .

The propositions related to the value discipline of a blockchain application, its implementation, and use have a direct influence on the growth versus profitability tension. In particular, a possible coping strategy lies in the question of whether a blockchain solution should primarily satisfy existing demand and requirements of a company's (internal or external) customers or go beyond, e.g., by offering a completely new solution path. Going beyond customer-centricity thereby might be a valuable approach, considering that the increasing amount of data available about customers and, thus, also participants and contributors of a blockchain, might be reduced to data points that only serve as means to an end to a blockchain provider (e.g., given a private blockchain) [56]. Avoiding the negative effects on customers and various stakeholders in a private blockchain, but also for blockchain ecosystems, considering going beyond customer-centricity helps to not turn against the customer by utilizing the massive amount of data available for customization [56]. At the same time, novel solutions for building trust through e.g., data passports for identity self-management that might foster growth and network effects [57]. Eventually, the general tension of growth vs. profitability is influenced by the blockchain-related tension of environmental positioning. Design decisions concerning the degree of openness and decentralization, therefore, need to be carefully considered to avoid undesired path dependencies. Also, companies might consider situational approaches, where, depending on the priority between growth and profitability push, they can adjust participation and validation rules [58].

Tension area 2 - Long term vs. short term: Dodd and Favaro [1] emphasize that companies tend to swing between a focus on strategy and execution. When focusing on strategy, typically companies were eager to concentrate on building for the future, leading to issues concerning performance today (e.g., difficulties in hitting earning targets) and vice versa. Likely, companies that want or are using blockchain will have to manage such tensions. These tensions are closely related to the controllable tension of organizational alignment and the manageable tensions of the integration model and the accountability model.

While the tension of long versus short term is reflected in the discussion around growth versus viability, short versus long term tensions require decision making on management level rather than business model level [1]. Above all, it shows that leaders need to rethink the companies' targets, processes, and routines, the former being reflected by the blockchainrelated tension of organizational alignment.

When it comes to organizational alignment, companies need to consider that in the short term, the implementation of private blockchains can work faster compared to open blockchains (since, among others, the logic of decision making in private blockchains is resembling the common business logic) [24]. Private blockchains, thus, can be faster integrated into organizational processes and routines (compared to fully open blockchains), thereby increasing the efficiency not only of a process as such but also their traceability and manageability, e.g., by providing full transparency on validation and log entries captured on a blockchain. On the other hand, organizations that want to exploit external value networks might need to consider finding appropriate strategic partners that support joint value creation, strategy, and shared corporate values. As Dodd and Favaro [1] pointed out, one 'middle ground' to control this tension is to focus on sustainable earnings, which is closely related to the integration and accountability model in blockchain.

The integration model regulates which access certain user groups have to data and verification mechanisms of the blockchain. Thus, this is a part of value sharing. While short-term perspective focuses on exercising control over the data and rights of a blockchain - thus control over value creation - a longterm strategy asks how values can be safeguarded when blockchain fosters inclusivity. Companies aiming to achieve sustainable earnings need to balance those two aspects, especially in open network environments characterized by shared operational efficiency [34].

Finally, the alignment along the extreme propositions of the accountability model determines the extent to which long-term strategic planning aspects are preferred over current revenues and a short-term alignment of the organization. Here, an alignment of the management is required with regard on the propositions of complete transparency versus anonymity in the blockchain network. In the short term, the former should be used to optimize internal processes by, among other things, making activities traceable, while at the same time promoting the flow of data within the company through an internal, private, or partially private blockchain. Through this, reliance on the profitability and yield of processes can be achieved.

On the other hand, the long-term orientation of the accountability model theoretically leads to control losses due to the anonymity of users and increased risks of data misuse and manipulation within the scope of cross-company processes [59]. However, since great risk often goes together with greater reward, it makes 
sense to relax strict accountability rules (if a company's industry and organizational structure allow, e.g., taking into account regulatory requirements) and encourage growth and shift to future performance indicators through larger networks (pseudo)anonymity. Here, the participation of different user groups can lead to distributed and combinatorial innovation in networks through different affordances, i.e., action potentials offered by a blockchain to participants and their interaction via the infrastructure [3].

Tension area 3 - Whole versus parts: Most companies are concerned with the tension that is concerned with whole versus parts, or centralization versus decentralization (note that for instance, public blockchain might be distributed, but at the beginning not yet decentralized until a critical mass of participants is reached). While typically swinging between those propositions, Dodd and Favaro [1] indicate that discussions to determine the degree of (de)centralization revolve around the exploitation of synergies, which can only be exploited by focusing on the whole, while at the same time, influencing and limiting the individual business units, thus leading to eroding profits, which, in turn, leads to a focus on parts of the organization, e.g., certain corporate departments, units, or teams [1].

Overall, these alignments have close relation with corporate culture and cultural identity. Strict cultural identities across business units or functions promote a focus on parts of the company, i.e., decentralization. Thus, in this tension, business decisions revolve around the organizational model, i.e., structures, culture, and people, with trust being one aspect of these aspects.

The trust model must be carefully selected in the context of a blockchain project, as it is the crux of any blockchain, resulting in intermediation and the potential reduction, if not elimination, of intermediaries and transaction costs [60]. Thus, many of the technology's value propositions are realized through the trust model. Companies should consider whether, when using blockchain as a intra-corporate (niche) solution, there is the possibility of interpersonal or institutional trust, to emerge. This would make a technical solution through the blockchain virtually superfluous for trust-building the blockchain would then be an infrastructure for processing data. In contrast, a focus on the entire company, i.e., the whole, is often accompanied by a more extensive solution, which might require a technology-based trust model, e.g., between business units of companies that cooperate via a blockchain.

The common bond to deal with tensions related to the whole versus parts is what [1] calls diagonal assets, which are particularly organizational resources and capabilities that help the company act as both a single company and many different businesses at the same time. This consideration can be discussed in the context of the trust model and under consideration of the aspects for which corporate purposes a blockchain with algorithmic trust should be used. In other cases, a blockchain might need to be interoperable with other (already existing) information systems to focus on the parts. Finally, another aspect of the design could be the use of a combination of 'trustless' on-chain and trustworthy off-chain storage, which at the same time makes it possible to protect corporate resources in the sense of storage capacities [61].

\section{Conclusion and Outlook}

This paper presents a structured literature review that identifies tensions in the implementation and use of blockchain technology.

Scientific contribution: Scholars have studied tensions in the implementation and use of blockchain to contribute to the successful use of the decentralized technology. While this literature covers many use cases of the technology (e.g., in blockchain in logistics or commerce), it is apparent that hitherto the identified tensions are case-specific and, therefore, neither fully transferable to other cases, nor generalizable due to context-sensitivity of the identified tensions.

This paper contributes to the current body of literature by addressing this research gap. It compiles blockchain-related tensions and analyses these on a more abstract level so that researchers can systematically think through blockchain-related tensions and corresponding design decisions. This might assist researchers, for example, when developing new use cases and blockchain-based prototypes. By discussing and interpreting blockchain-specific tensions in the context of organizations' overall tensions (e.g., growth vs. profitability), the framework also helps researchers in better anticipating the long-term, potentially unintended effects of blockchain implementation and use on various technical, organizational, and cultural aspects of an organization.

Thereby, the framework is also intended to help companies, as it provides initial food for thought on how to address tensions in blockchain and thus represents a first step in developing recommendations for addressing them. We plan to refine the framework and apply it to companies that have developed, want to develop, or have failed to develop blockchain prototypes to derive recommendations for these cases.

These considerations are directly related to the limitations of this paper. The framework and the questions regarding the control and management of tensions in the implementation and use of the blockchain are currently still in a theoretical-conceptual stage, resulting from the inductive approach of deriving the tensions from the existing IS literature. This 
limitation is to be solved in the course of the further development of the study, in particular by (1) extending the literature search to databases of other research fields, i.e., management and computer science, and (2) by adding case studies and interviews with industry experts, to examine the validity of the framework. Only by addressing the current data bottleneck necessary progress towards the advancement of the framework and the questions posed in the context of the three major areas of tension of an organization can be made. Further, insights gained in the discussion are based on the general tensions every company faces [1]. While on the one hand, this shows the role and relevance of the tensions in these areas of consideration for companies, the resulting high degree of abstraction is another limitation that needs to be addressed in the future.

\section{References}

[1] Dodd D, Favaro K. Managing the Right Tension. Harv Bus Rev 2006:1-15.

[2] Groen AJ, Wakkee IAM, Weerd-Nederhof PC. Managing Tensions in a High-Tech Start-Up; An Innovation Journey in Social System Perspective. Int Small Bus J 2008;26:57-81.

[3] Ostern NK, Rosemann M, Moormann J. Determining the Idiosyncrasy of Blockchain: An Affordances Perspective. 41st Int. Conf. Inf. Syst., 2020, p. 1-17.

[4] Abbatemarco N, De Rossi LM, Aakanksha G, Salviotti G. Beyond a Blockchain Paradox: How Intermediaries Can Leverage a Disintermediation Technology. Hawaii Int. Conf. Syst. Sci. (HICSS 2020), 2020, p. 1-10.

[5] Tumasjan A, Beutel T. Blockchain-based decentralized business models in the sharing economy: A technology adoption perspective. Bus. Transform. Through Blockchain, 2019, p. 77-120.

[6] Dumas M, Hull R, Mendling J, Weber I. Blockchain Technology for Collaborative Information Systems. Dagstuhl Reports, 2019, p. 67-129.

[7] Schmeiss J, Hoelzle K, Tech RPG. Designing Governance Mechanisms in Platform Ecosystems: Addressing the Paradox of Openness Through Blockchain Technology. Calif Manage Rev 2019;62:121-43.

[8] Furlonger D, Uzureau C. The Five Kinds of Blockchain Projects. Harv Bus Rev 2019.

[9] Beck R, Avital M, Rossi M, Thatcher JB. Blockchain Technology in Business and Information Systems Research. Bus Inf Syst Eng 2017;59:381-4.

[10] Rossi M, Mueller-Bloch C, Thatcher JB, Beck R. Blockchain Research in Information Systems: Current Trends and an Inclusive Future Research Agenda. J Assoc Inf Syst 2019;20:247-65.

[11] Beck R, Müller-Bloch C, King JL. Governance in the Blockchain Economy: Framework and Research Agenda. J Assoc Inf Syst 2018;19:1020-34.

[12] Uchibeke UU, Schneider KA, Kassani SH, Deters
R. Blockchain access control Ecosystem for Big Data security. IEEE Smart Data, 2018, p. 1373-8.

[13] van Hoek R. Exploring blockchain implementation in the supply chain: Learning from pioneers and RFID research. Int J Oper Prod Manag 2019;39:829-59.

[14] Coblenz M, Hull R, Lu Q, Weber I. A Holistic Vision of Blockchain-Based Application Design, Specification, and Implementation. Dagstuhl Reports, 2019, p. 90-106.

[15] Zavalokina L, Ziolkoswki R, Bauer I, Schwabe G. Management, Governance and Value Creation in a Blockchain Consortium. MIS Q Exec 2020;19:1-17.

[16] Ciriello RF, Beck R, Thatcher JB. The Paradoxical Effects of Blockchain Technology. Int. Conf. Inf. Syst., 2018, p. 1-17.

[17] Andriopoulos C, Lewis MW. ExploitationExploration Tensions and Organizational Ambidexterity: Managing Paradoxes of Innovation. Organ Sci 2008;20:685-834.

[18] Wolfswinkel JF, Furtmueller E, Wilderom CPM. Using grounded theory as a method for rigorously reviewing literature. Eur J Inf Syst 2013;22:45-55.

[19] Gläser J, Laudel G. Life with and without coding: Two methods for early-stage data analysis in qualitative research aiming at causal explanations. Forum Qual Sozialforsch 2013;14:1-25.

[20] Syed M, Nelson SC. Guidelines for Establishing Reliability When Coding Narrative Data. Emerg Adulthood 2015;3:375-87.

[21] Bazarhanova A, Magnusson J, Lindman J, Chou E, Nilsson A. Blockchain-based electronic identification: cross-country comparison of six design choices. Eur. Conf. Inf. Syst. (ECIS 2019), 2019, p. 1-16.

[22] Norstrom L, Lindman J. Exploring Blockchain Municipal Use Cases. Proc. Am. Conf. Inf. Syst. (AMCIS 2020), 2020, p. 1-10.

[23] Hoess A, Schlatt V, Rieger A, Gilbert F. The Blockchain Effect: From Inter-Ecosystem to IntraEcosystem Competition. 29th Eur. Conf. Inf. Syst., 2021, p. 1-16.

[24] Viriyasitavat W, Hoonsopon D. Blockchain characteristics and consensus in modern business processes. J Ind Inf Integr 2019;13:32-9.

[25] Berente N, Lyytinen K, Yoo Y, King JL. Routines as Shock Absorbers During Organizational Transformation: Integration, Control, and NASA's Enterprise Information System. Organ Sci 2016;27:551-72.

[26] Ciborra C, Braa K, Cordella A, Hepsø V, Dahlbom B, Failla A, et al. From control to drift: The dynamics of corporate information infrastructures. Oxford University Press (on demand); 2000.

[27] Gordon W, Catalini C. Blockchain technology for healthcare: facilitating the transition to patientdriven interoperability. Comput Struct Biotechnol J 2018;16:224-30.

[28] Bogusz CI, Morisse M. How infrastructures anchor open entrepreneurship: The case of Bitcoin and stigma. Inf Syst J 2018;39:1176-212. 
[29] Renwick R, Gleasure R. Those who control the code control the rules: How different persepctives of privacy are being written into the code of blockchain systems. J Inf Technol 2021;36:16-38.

[30] Schweizer A, Schlatt V, Urbach N, Fridgen G. Unchaining Social Businesses - Blockchain as the Basic Technology of a Crowdlending Platform. ICIS Proc., 2017.

[31] Villareal B, Garza F, Rosas I, Garcia D. An Introduction to Distribution Operational Efficiency. Int J Ind Eng 2012;19:278-88.

[32] Chen Y, Pereira I, Patel PC. Decentralized governance of digital platforms. J Manage 2021;47:1305-37.

[33] Iansiti M, Lakhani KR. The truth about Blockchain: Harv Bus Rev 2017.

[34] Bauer I, Zavolokina L, Leisibach F, Schwabe G. Exploring the Blockchain Value Creation: The Case of the Car Ecosystem. 52nd Hawaii Int. Conf. Syst. Sci., 2018, p. 1-10.

[35] Holotiuk F, Moormann J. Organizational Adoption of Digital Innovation. 26th Eur. Conf. Inf. Syst. 2018, p. 1-16.

[36] Morkunas V, Paschen J, Boon E. How blockchain technologies impact your business model. Bus Horiz 2019;62:295-306.

[37] Montealegre R, Iyengar K, Sweeney J. Understanding Ambidexterity: Managing Contradictory Tensions Between Exploration and Exploitation in the Evolution of Digital Infrastructure. J Assoc Inf Syst 2019;20:647-80.

[38] Böhme R, Christin N, Edelman B, Moore T. Bitcoin: Economics, Technology, and Governance. J Econ Perspect 2015.

https://doi.org/10.1257/jep.29.2.213.

[39] Naerland K, Müller-Bloch C, Beck R, Palmund S. Blockchain to Rule the Waves - Nascent Design Principles for Reducing Risk and Uncertainty in Decentralized Environments. Proc. Int. Conf. Inf. Syst., 2017.

[40] Perscheid G, Ostern N, Moormann J. Determining Platform Governance: Framework for Classifying Governance Types. 41 st Int. Conf. Inf. Syst., Hyderabad, India: 2020, p. 1-16.

[41] Beck R, Czepluch JS, Lollike N, Malone S. Blockchain - the Gateway To Trust- Free Cryptographic Transactions. Twenty-Fourth Eur Conf Inf Syst 2016.

[42] Zhao L, Fan S, Zheng E. Blockchain enabled trust: the case of inter-firm dataflow. Proceeding 24th Am. Conf. Inf. Syst., 2018, p. 1-10.

[43] Glaser F, Hawlitschek F, Notheisen B. Blockchain as a Platform. Bus. Transform. Through Blockchain, vol. 1, Palgrave Macmillan; 2019.

[44] Auinger A, Riedl R. Blockchain and Trust: Refuting Some Widely-Held Misconceptions. Int. Conf. Inf. Syst. (ICIS 2018), 2018, p. 1-17.

[45] Betzwieser B, Franzbonenkamp S, Riasanow T, Klenegger H, Krcmar H. A Decision Model for the Implementation of Blockchain Solutions. 25th Am. Conf. Inf. Syst., 2019, p. 1-10.
[46] Ostern NK. Do You Trust a Trust-Free Technology? Toward a Trust Framework Model for Blockchain Technology. Int. Conf. Inf. Syst., 2018, p. 1-17.

[47] Andersen JV, Ingram Bogusz C. Patterns of SelfOrganising in the Bitcoin Online Community : Code Forking as Organising in Digital Infrastructure. Proc. $38^{\text {th }}$ Int. Conf. Inf. Syst. (ICIS 2017), 2017.

[48] Currie WL, Gozman DP, Seddon JM. Dialectic Tensions in the Financial Markets: A Longitudinal Study of Pre- and Post-Crisis Regulatory Technology. J Inf Technol 2019;33.

[49] Yin HHS, Langenheldt K, Harlev M, Mukkamala RR, Vatrapu R. Regulating Cryptocurrencies: A Supervised Machine Learning Appraoch to DeAnonymizing the Bitcoin Blockchain. J Manag Inf Syst 2019;36:37-73.

[50] Reid F, Harrigan M. An analysis of anonymity in the bitcoin system. Secur. Priv. Soc. Networks, 2013.

[51] Fabian B, Ermakova T, Sander U. Anonymity in bitcoin - The users' perspective. ICIS 2016 Proc., 2016.

[52] Scholl HJ, Pomeshchikov R, Rodriguez MPB. Early Regulations of Distributed Ledger Technology/Blockchain Providers: A Comparative Case Study. Proc. 53rd Hawaii Int. Conf. Syst. Sci., 2020, p. 1760-9.

[53] Ostern NK, Riedel J. Know-Your-Customer (KYC) Requirements ofr Intial Coin Offerings. Bus Inf Syst Eng 2020:1-17.

[54] Rieger A, Guggenmos F, Lockl J, Fridgen G, Urbach N. Building a Blockchain Application that Complies with the EU General Data Protection Regulation. MIS Q Exec 2019;18:263-79.

[55] Müller M, Ostern N, Rosemann M. Silver Bullet for All Trust Issues? Blockchain-Based Trust Patterns for Collaborative Business Processes. Bus. Process Manag. (BPM 2020), Seville, Spain: 2020, p. 13-8.

[56] Riedmann-Streitz. Redefining the Customer Centricity Approach in the Digital Age. Int. Conf. Des. User Exp. Usability, 2018, p. 203-22.

[57] Ølnes S, Ubacht J, Janssen M. Blockchain in government: Benefits and implications of distributed ledger technology for information sharing. Gov Inf Q 2017;34:355-64.

[58] Wan C, Tang S, Zhang Y, Pan C, Liu Z, Long Y, et al. Goshawk: A Novel Efficient, Robust and Flexible Blockchain Protocol. Int. Conf. Inf. Secur. Cryptol., 2018, p. 49-69.

[59] Cumming DJ, Johan S, Pant A. Regulation of the Crypto-Economy: Managing Risks, Challenges, and Regulatory Uncertainty. J Risk Financ Manag 2019;12:126-40.

[60] De Filippi P, Mannan M, Reijers W. Blockchain as a confidence machine: The problem of trust \& challenges of governance. Technol Soc 2020;62:114.

[61] Al-Breiki H, Rehman MHU, Salah K, Sventinovic D. Trustworthy Blockchain Oracles: Review, Comparison, and Open Research Challenges. IEEE Acess 2020;8:85678-85. 